\title{
Phase field simulation of liquid-solid-eutectoid multiple phase transformations of a Fe-C binary alloy
}

\author{
*Li Feng', 2, Jun-he Zhong', Chang-sheng Zhu'2, Jun Wang', Guo-sheng An ${ }^{1,2}$, Rong-zhen Xiao ${ }^{1,2}$ \\ 1. College of Materials and Engineering, Lanzhou University of Technology, Lanzhou 730050, China \\ 2. State Key Laboratory of Advanced Processing and Recycling of Non-ferrous Metals, Lanzhou University of Technology, \\ Lanzhou 730050, China
}

\begin{abstract}
A new continuous multi-phase transformation field model was established for liquid-solid-eutectoid transformation. Taking Fe-C alloy as an example, the model was used to simulate the evolution of the micromorphology of the liquid-solid phase transition, and the effects of temperature, solute and free energy on the nucleation of pearlite after the liquid-solid phase transition were analyzed. The micro-morphology of pearlite was simulated. The simulation results show that the austenite structure has hereditary effect on the pearlite, the morphology of pearlite structure was similar to that of the parent austenite. The eutectoid structure at the front of pearlite grows toward the interior of austenite grains in a bifurcation manner and in the spherical coronal shape. In addition, the growth rate of pearlite was related to the shape of concave-convex interface at the nucleation site, and the growth rate at the convex interface was faster than that the concave interface.
\end{abstract}

Key words: multi-phase field method; multiple phase transformation; pearlite; free energy; numerical simulation CLC numbers: TP391.9

Document code: $\mathrm{A}$

Article ID: 1672-6421(2020)04-301-07

$\mathrm{P}$ hase transformation in metal solidification process is a complex process involving heat transfer at high temperature, convection, solute diffusion and interaction between metal and mold ${ }^{[1]}$. In addition, the metal is opaque and the phase transformation process cannot be observed directly, which brings difficulties to research ${ }^{[2]}$. By using numerical simulation, the evolution of complex phase transformation morphology during solidification can be directly observed. Phase field method is one of the commonly used methods to study the microstructure evolution of phase transformation of alloys. It describes the microstructures and compositions of grain structure by a series of field variables. The advantage of phase field method is that it is not necessary to track the complex solid/liquid interface, and easy to be coupled with other external fields. Phase field method can accurately simulate the solid-liquid interface migration and the morphology evolution during the phase transformation process; it can also quantitatively analysis the influence

\section{*Li Feng}

Male, born in 1981, Associate Professor. His research interests mainly focus on the numerical simulation of microstructure in solidification process.

E-mail: fenglils@lut.edu.cn

Received: 2019-11-05; Accepted: 2020-04-13 of disturbance, anisotropy and supercooling degree on microstructure and predict the phase transformation process in materials ${ }^{[3]}$.

At present, most of the studies on the microstructural evolution of alloys during phase transformation by phase field method mainly focus on the single process of liquid-solid or solid-solid phase transformation. For the phase-field simulation of liquid-solid phase transformation, Kang et al. ${ }^{[4]}$ simulated dendrite growth during directional solidification of $\mathrm{Fe}-\mathrm{C}$ binary alloy by phase field method, studied the effects of anisotropy, temperature gradient and advancing speed on dendrite morphology. Tourret and Karma ${ }^{[5]}$ studied the effect of temperature gradient on the morphology of columnar crystals by simulating the competitive growth of two-dimensional directional solidification columnar crystals by phase field method. Feng et al. ${ }^{[6]}$ studied the influence of grain orientation angle and axial angle on the growth morphology of columnar crystals by simulating the competitive growth of columnar crystals during three-dimensional solidification of $\mathrm{Al}-\mathrm{Cu}$ binary alloy based on phase field method. Zhu et al. ${ }^{[7]}$ simulated the dendritic growth and recrystallization of $\mathrm{Ni}-\mathrm{Cu}$ binary alloy during the solidification at different cooling rates, and studied the effects of cooling rate on dendritic patterns. Zhang et al. ${ }^{[8,9]}$, based on the phase 
field method, simulated the dendrite growth and the lamellar eutectic growth of Al-Cu alloy. In the study of solid-solid phase transformation, Zhang et al. ${ }^{[10]}$ established the nucleation model of solid-solid phase transformation based on phase field method. The morphology and growth kinetics of ferrite grains under different grain boundary characteristics during austenite-ferrite transformation of $\mathrm{Fe}-\mathrm{C}$ alloy were simulated. Based on this work, they further simulated the transformation of austenite-ferrite during the isothermal process of Fe-C-Mn ternary alloy ${ }^{[1]}$, and the influence of Mn content on microstructure and transformation kinetics of austenite-ferrite transformation was studied. Nakajima et al. ${ }^{[12]}$ simulated the synergy growth of pearlite in eutectoid steel, and studied the effect of supercooling degree on lamellar spacing and growth rate.

While, in the process of solidification, such as the Fe-C alloys, continuous phase transformation process of liquid-solid to solid-solid often occurs. A single simulation of liquid-solid or solid-solid phase transformation process cannot accurately characterize the solidification process and state of the alloys. In this work, a new multi-phase field model for liquidsolid-eutectoid multiple phase continuous transformation is established by coupling liquid-solid phase transformation and eutectoid phase transformation. Taking the Fe-C binary alloy as an example, the microstructure evolution process of continuous phase transformation of the alloy is simulated.

\section{Multi-phase field model}

\subsection{Phase field control equation}

The continuous multi-phase transformation field model was coupled by the governing equation of the liquid-solid and the eutectoid phase transition. The sequential function $K$ is used as the sequential control term of continuous phase transformation ${ }^{[13]}$. The governing equation of phase field is as follows:

$$
\frac{\partial \phi_{i}}{\partial t}=\sum_{\alpha \neq \beta}^{m} K_{\alpha \beta} \cdot \frac{2}{n} \sum_{j \neq i}^{n} S_{i j} M_{i j}\left(\frac{\delta F}{\delta \phi_{i}}-\frac{\delta F}{\delta \phi_{j}}\right)
$$

where, $\phi_{i}$ and $\phi_{j}$ are the phase field parameters of different phase structures, $\phi_{i}=1$ in the phase $i$, and $\phi_{i}=0$ in other phases, $\phi_{j}=1$ in the phase $j$, and $\phi_{j}=0$ in other phases, $M_{i j}$ is the interface migration rate, $S_{i j}$ is the space step function ${ }^{[14]}, m$ is the number of times of phase transformations, $F$ is the system free energy, $n$ is the number of phase field variables, $n=3$. The subscripts $i$ and $j$, respectively, represent the new phase and parent phase in the liquid-solid phase transformation and eutectoid transformation. The subscripts $\alpha$ and $\beta$ represent the liquid-solid phase transformation and eutectoid transformation, respectively. The definition of the sequential function $K$ is as follows:

$$
\begin{array}{ll}
K_{\alpha}\left(T_{t}^{\mathrm{x}}, f^{i}\right)=1 & \left(T_{t}^{\mathrm{x}} \leq T_{i}^{\mathrm{e}}\right) \\
K_{\alpha}\left(T_{t}^{\mathrm{x}}, f^{i}\right)=0 & \left(T_{t}^{\mathrm{x}}>T_{i}^{\mathrm{e}}\right)
\end{array}
$$

where, $t$ is time, $T^{\mathrm{x}}$ represents the system temperature, and $T^{\mathrm{e}}$ represents the temperature at the beginning of the phase transformation, $f^{i}$ represents the component free energy of solute in phase $i$. The free energy $F$ of the system is specifically defined as follows:

$$
F=\int_{\Omega} f^{\mathrm{CH}}+f^{T}+f^{\mathrm{EL}}+f^{\mathrm{GB}}
$$

where, $f^{\mathrm{GB}}, f^{\mathrm{CH}}$, and $f^{\mathrm{EL}}$ denote the interfacial, component, and elastic free energy densities, respectively; $f^{T}$ represents free energy density relating to a specific temperature, $\Omega$ is an integral of the density functional over the domain ${ }^{[15]}$. The expressions are as follows:

$$
\begin{gathered}
f^{\mathrm{CH}}=\sum_{i=1}^{N} \phi_{i} f^{i}\left(c_{i}\right) \\
f^{T}=h(\phi) G_{i}^{\mathrm{reg}}+(1-h(\phi)) G_{j}^{\mathrm{reg}}+W g\left(\phi_{i}\right) \\
f^{\mathrm{EL}}=\frac{1}{2}\left(\varepsilon^{i j}-\varepsilon^{* i j}\right) C^{i j k l}\left(\varepsilon^{k l}-\varepsilon^{* k l}\right) \\
f^{\mathrm{GB}}=\sum_{i, j=1}^{\mathrm{N}} \frac{4 \sigma_{i j}}{\eta_{i j}}\left\{\frac{\eta_{i j}^{2}}{\pi^{2}}\left|\nabla \phi_{i} \cdot \nabla \phi_{j}\right|+W_{i j}\right\}
\end{gathered}
$$

where, $f^{i}\left(c_{i}\right), C^{i j k l}, \varepsilon, \eta_{i j}, \sigma_{i j}$, and $W_{i j}$ represent the component free energy of solute $c$ in phase $i$, the effective elasticity matrix, the phase-field gradient coefficient, interfacial thickness, interfacial energy, and potential-well function, respectively. In addition,the effective eigenstrain $\varepsilon^{*_{i j}}$ and the effective elasticity matrix $C^{i j k l}$ were introduced. $G^{\text {reg }}$ denotes the Gibbs free energy, $G^{\mathrm{reg}}=\sum_{i=1}^{2}\left(c_{i} \mu_{i}^{0}+R T c_{i} \ln c_{i}\right)+\sum^{2} c_{i} c_{j} \sum^{n}\left(c_{i}-c_{j}\right)$, and $\operatorname{Wg}\left(\phi_{i}\right)$ is the excess free energy, $W=\phi_{i} \phi_{j}\left(0<\phi_{i}<1,0<\phi_{i}<1\right), g\left(\phi_{i}\right)=$ $\phi_{i}^{2}\left(\phi_{i}^{2}-2 \phi_{i}-1\right)$. To simplify the model, the influences of the stress and strain fields were neglected. Therefore, the free energy $F$ was changed to

$$
F=\int_{\Omega} f^{\mathrm{GB}}+f^{\mathrm{CH}}+f^{\mathrm{T}}
$$

\subsection{Solute control equation}

The governing equation of solute diffusion field is

$$
\frac{\partial c}{\partial \mathrm{t}}=\nabla\left[M_{\mathrm{C}} \nabla\left(\frac{\delta F}{\delta C}\right)\right]=\nabla\left[\frac{D(c, \phi)}{f_{\mathrm{cc}}} \nabla f_{\mathrm{c}}\right]
$$

where, $M_{c}$ is the field parameters, i.e., interface mobility, $D(c, \phi)$ is the solute diffusion coefficient, $f_{\mathrm{c}}$ and $f_{\mathrm{cc}}$ are the first-order and second-order partial differential equations of free energy density:

$$
\begin{gathered}
f_{\mathrm{c}}(c, \phi)=\frac{d f^{i}\left(c_{i}\right)}{d c_{i}}=\frac{d f^{j}\left(c_{j}\right)}{d c_{j}} \\
f_{\mathrm{cc}}(c, \phi)=\frac{f_{\mathrm{cc}}^{i}\left(c_{i}\right) \cdot f_{\mathrm{cc}}^{j}\left(c_{j}\right)}{[1-h(\phi)] f_{\mathrm{cc}}^{j}\left(c_{j}\right)+h(\phi) f_{\mathrm{cc}}^{i}\left(c_{i}\right)}
\end{gathered}
$$

where, the subscripts $i$ and $j$ represent the new phase and 
parent phase in the solidification phase transformation or solidstate transformation, respectively. The $h(\phi)$ is the potential function, $h(\phi)=\phi^{3}\left(6 \phi^{2}-15 \phi+10\right)$.

\subsection{Temperature control equation}

The governing equation of temperature field is

$$
\begin{aligned}
\frac{\partial T}{\partial t}= & D_{T} \cdot \nabla^{2} T-\frac{L(1-c)+L^{\prime} c}{C_{\mathrm{P}}} h^{\prime}(\phi) \frac{\partial \phi}{\partial t}- \\
& \frac{L-L^{\prime}}{C_{\mathrm{P}}}\left[1-h^{\prime}(\phi)\right] \frac{\partial c}{\partial t}
\end{aligned}
$$

where $D_{T}, C_{\mathrm{P}}, L, L^{\prime}$, and $T$ represent the thermal diffusion coefficient, specific heat, latent heat of the solvent, latent heat of the solute, and temperature, respectively ${ }^{[12]}$.

\subsection{Phase field parameters}

The expression of the migration rate $M_{\mathrm{L}}$ of the liquid-solid phase transformation is

$$
\begin{gathered}
M_{\mathrm{L}}^{-1}=\frac{\varepsilon_{0}^{3}}{\sigma \sqrt{2 W}}\left(\frac{1}{D} \zeta\left(c_{\mathrm{L}}^{\mathrm{e}}, c_{\mathrm{S}}^{\mathrm{e}}\right)\right) \\
\varepsilon_{0}=\sqrt{\frac{6 \lambda \sigma}{2.2}} \\
W=\frac{6.6 \sigma}{\lambda}
\end{gathered}
$$

For solid-solid phase transformation, the expression of the migration rate $M_{i j}$ is

$$
\begin{gathered}
M_{i 3}=\frac{V_{\mathrm{m}}}{R T} \frac{\sigma_{i 3}}{\left(1-k_{i}\right)^{2}} \frac{\sqrt{2 \lambda_{i 3}}}{c_{i 3}^{\mathrm{e}} \varepsilon_{i 3}^{\mathrm{e}} \zeta_{i 3}^{\mathrm{e}}} \\
\varepsilon_{i j}=\frac{4}{\pi} \sqrt{\zeta \sigma_{i j}}
\end{gathered}
$$

where, $\varepsilon, \lambda$, and $\sigma$ are gradient energy coefficient, interface thickness, and interface energy, respectively, and $\lambda=2.5 \Delta x$, $\zeta=0.5 \lambda$.

\subsection{Nucleation model}

The nucleation region of solid-solid transformation is in the place where the free energy of the local system is higher, generally at the grain boundary of parent phase, the area where solute segregation is serious and where the stress and strain are concentrated. Therefore, the morphology of solidified parent phase, grain boundary energy, local system free energy, solute distribution, and nucleation density should be considered in the model of solid-solid transformation nucleation. The morphology of solidified parent phase can be calculated by the phase field governing Eq. 1. The influence of austenite grain boundary energy on the local system free energy during the liquid-solid transformation can be calculated by Eq. 8 . The local system free energy can be calculated by Eq. 9, and the solute distribution can be obtained by Eq. 10. The nucleation density should conform to Gaussian distribution, as follows:

$$
\frac{d n}{d(\Delta t)}=\frac{n_{\max }}{\sqrt{2 \pi} \Delta T_{\sigma}} \exp \left(-\frac{\left(\Delta T-\Delta T_{\max }\right)^{2}}{2 \Delta T_{\sigma}^{2}}\right)
$$

where, $n_{\max }, \Delta T_{\max }$, and $\Delta T_{\sigma}$ represent the maximum nucleation density, maximum nucleation undercooling degree, and

\begin{tabular}{|c|c|}
\hline Parameter & Fe-C alloy \\
\hline $\begin{array}{c}\text { Solid-liquid and solid-solid interface } \\
\text { energy, } \sigma\end{array}$ & $0.204 \mathrm{~J} \cdot \mathrm{m}^{-2}, 1 \mathrm{~J} \cdot \mathrm{m}^{-2}$ \\
\hline $\begin{array}{c}\text { Melting point and phase transition } \\
\text { temperature, } T_{\mathrm{m}}, T_{\mathrm{f}}\end{array}$ & $1,801 \mathrm{~K}, 1,048 \mathrm{~K}$ \\
\hline $\begin{array}{l}\text { Liquid phase and solid phase } \\
\text { solute diffusivity, } D_{\mathrm{L}}, D_{\mathrm{S}}\end{array}$ & $\begin{array}{l}2.0 \times 10^{-8} \mathrm{~m}^{2} \cdot \mathrm{s}^{-1} \\
6.0 \times 10^{-9} \mathrm{~m}^{2} \cdot \mathrm{s}^{-1}\end{array}$ \\
\hline $\begin{array}{l}\text { Austenite and ferrite diffusion rate, } \\
\qquad D_{1}, D_{2}\end{array}$ & $\begin{array}{l}1.0145 \times 10^{-12} \mathrm{~m}^{2} \cdot \mathrm{s}^{-1} \\
2.2435 \times 10^{-11} \mathrm{~m}^{2} \cdot \mathrm{s}^{-1}\end{array}$ \\
\hline Gas constant, $R$ & $8.314 \mathrm{~J} \cdot(\text { mole } \cdot \mathrm{K})^{-1}$ \\
\hline Latent heat of the solvent, $L$ & $270 \mathrm{~J} \cdot \mathrm{g}^{-1}$ \\
\hline Thermal diffusion coefficient, $D_{\mathrm{T}}$ & $1.277 \times 10^{-5} \mathrm{~J} \cdot \mathrm{g}^{-1}$ \\
\hline $\begin{array}{c}\text { Specific heat capacity at constant } \\
\text { pressure, } C_{\mathrm{P}}\end{array}$ & $0.75 \mathrm{~J} \cdot(\mathrm{g} \cdot \mathrm{K})^{-1}$ \\
\hline Molar volume, $V_{\mathrm{m}}$ & $7.7 \times 10^{-6} \mathrm{~m}^{3} \cdot \mathrm{mole}^{-1}$ \\
\hline$\Delta t_{1}, \Delta t_{2}$ & $3 \times 10^{-9} \mathrm{~s}, 1.7 \times 10^{-7} \mathrm{~s}$ \\
\hline
\end{tabular}
standard variance undercooling degree, respectively. All these parameters can be corrected by experimental observations.

\section{Numeral calculation of model}

\subsection{Physical parameters of material}

Table 1 lists the physical parameters of Fe-C binary alloy used in this study.

Table 1: Physical parameters of Fe-C binary alloy ${ }^{[13,15]}$

\subsection{Initial and boundary conditions}

The simulation calculation area was set as a grid of $1,200 \times 1,200$, the $\phi$ and $c$ values under the adiabatic boundary condition, i.e., a zero-Neumann boundary condition, were used. The nucleation is heterogeneous; the liquid-solid phase transition initial crystal nucleus radius is set to be $r=14 \times 10^{-8} \mathrm{~m}$, and the solid-solid phase transformation nucleation is set at the austenite grain boundary with a fixed lamellar width (the calculated lamellar width of ferrite and cementite is about 7:1). The finite difference method was used to solve the control equation in phase field model, the time step $\Delta t$ and the space step $\Delta x\left(\Delta x=\Delta y=1 \times 10^{-8} \mathrm{~m}\right)$ need to meet the following stability condition:

$$
\Delta t<\min \left\{\Delta x^{2} /\left(4 D_{t}\right), \Delta y^{2} /\left(4 D_{t}\right)\right\}
$$

In the process of multiple phase transition calculation, $\Delta t_{1}$ and $\Delta t_{2}$ denote the time steps of calculating the liquid-solid phase transformation and the eutectoid transformation, respectively. However, Fe-C alloy has a great temperature difference from liquid-solid to its eutectoid phase transformation, so a long 
cooling time is required. If small time steps are used, it calls for remarkable amounts of calculations. In order to facilitate the simulation, the solute diffusion was controlled in the process of continuous phase transformation by using the solute diffusion equation given by Fick's law with an enlarged time step $\Delta t_{12}$. In the transition period between the two-phase transformations, no phase transformation occurs, so the spatial step size of the simulation is no longer affected by the interface thickness in the phase transformation. Larger time step sizes and space step sizes can then be used to calculate the solute diffusion and temperature diffusion. So, $\Delta t_{12}$ was used to simulate the temperature, solute, and free energy diffusion in the transition period from the end of the liquid-solid transformation to the beginning of eutectoid transformation.

\section{Results and analysis}

\subsection{Simulation results and analysis of liquid- solid transformation}

In the simulation, the nucleus of liquid-solid phase transition is formed by the heterogeneous nucleation method. Five randomly distributed austenite grain nuclei are set in the calculation area, and the crystal growth is simulated after the initial temperature given. The growth morphology and solute distribution of austenite grains at an initial temperature of $1,678 \mathrm{~K}$ at different times are shown in Fig. 1. Figure 1a shows the austenite growth morphology at 30,000 $\Delta t_{1}$, the growth of continuous austenite grain can be obviously observed. Meanwhile, the grains grow independently and the dendrites have no contact with each other.

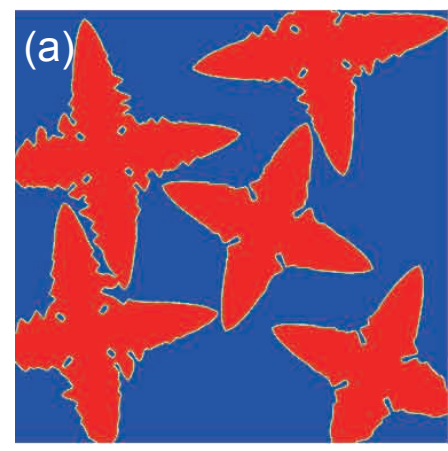

$30,000 \Delta t_{1}$

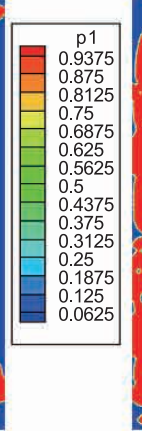

Fig. 1: Simulation results of morphology of austenite in liquid-solid phase transformation

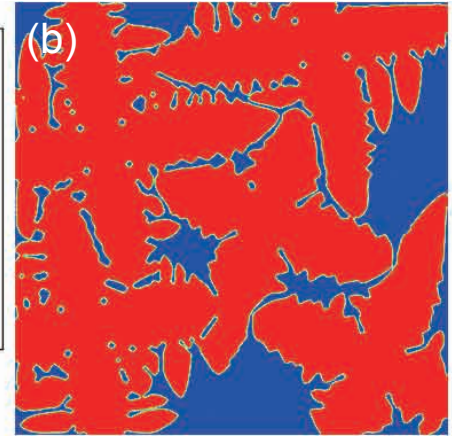

$50,000 \Delta t_{1}$
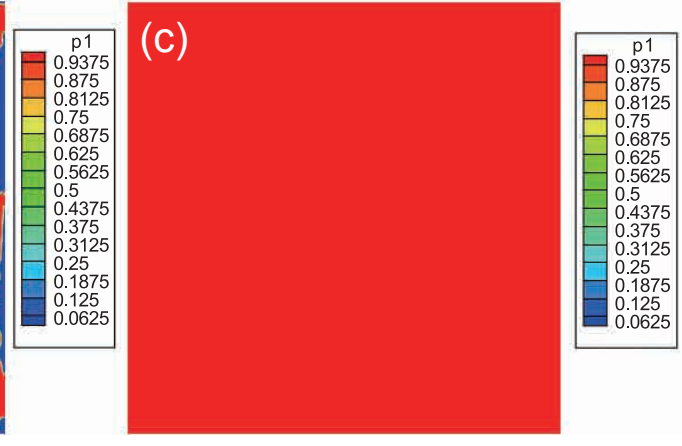

$70,000 \Delta t_{1}$
Figure 1(b) shows the austenite morphology at 50,000 $\Delta t_{1}$. With the formation and growth of the secondary and tertiary dendritic arms on the main dendrites, the grains contact each other. The secondary and tertiary dendritic arms are bent or stopped growing at the place where the dendritic arms come in contact. With the growth of grains, the whole simulation region is gradually filled with austenite at 70,000 $\Delta t_{1}$, as shown in Fig. 1(c).

The temperature is the key to control the continuous phase transformation in the process of multiple phase transformations. Figure 2 shows the simulation results of temperature field during liquid-solid phase transition. The initial temperature gradient in the simulation region is linear. As the cooling rate is ranged from $10^{-1} \mathrm{~K} \cdot \mathrm{s}^{-1}$ to $10 \mathrm{~K} \cdot \mathrm{s}^{-1}$ during solidification of medium and small castings at normal speeds ${ }^{[13]}$, a temperature gradient of $5 \mathrm{~K} \cdot \mathrm{mm}^{-1}$ at the boundaries was added to the model in this research. It is speculated that the heat is transferred from the bottom up. The temperature distribution results are shown from Fig. 2(a) to (c) at $0 \Delta t_{12}, 200,000 \Delta t_{12}$, and 400,000 $\Delta t_{12}$, respectively. As can be seen from Fig. 2, the simulation results show that the temperature of the solidified area is higher than the area not solidified. The solidification interface preface constantly releases latent heat which spreads from high temperature to low temperature. Larger time step can be used to calculate the temperature diffusion and the $\Delta t_{12}$ accelerates the calculation of temperature drop in the temperature field. As a result, the temperature condition for pearlite nucleation can be achieved faster.

When the austenite fills the entire simulation region, the solute is enriched at the grain boundary. At the same time, the diffusion is not uniform. Therefore, the enlarged time step $\Delta t_{12}$

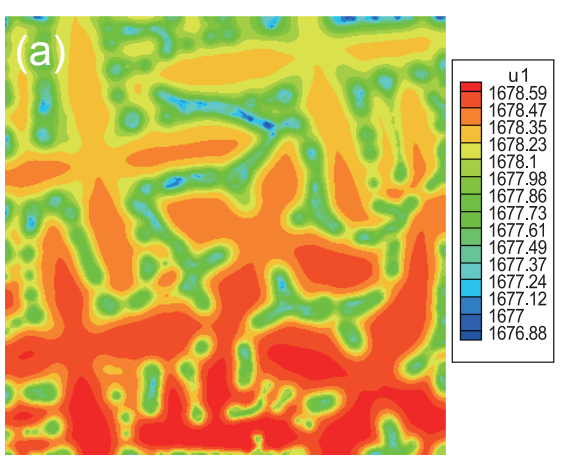

$0 \Delta t_{12}$

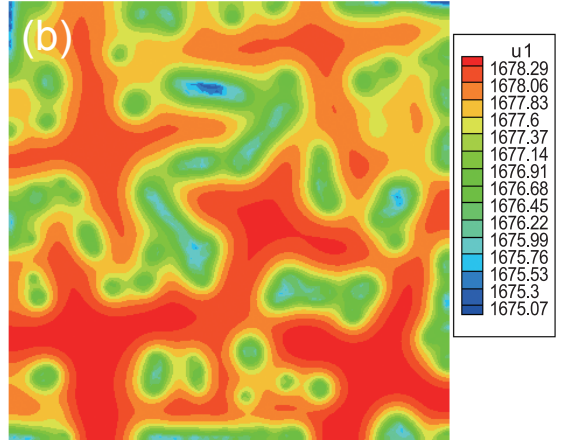

$200,000 \Delta t_{12}$

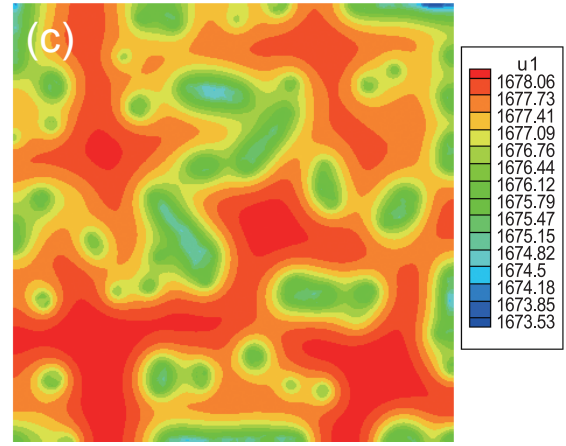

$400,000 \Delta t_{12}$

Fig. 2: Simulation results of temperature field during liquid-solid phase solidification 
is applied to the simulated region to diffuse the solute in the late solidification stage. The solute distribution results are shown in Figs. 3(a)-(c) at time of $0 \Delta t_{12}, 200,000 \Delta t_{12}$, and 400,000 $\Delta t_{12}$, respectively. As can be seen from Fig. 3 that the morphology of austenite does not change during solute diffusion, the diffusion of solute in the whole region is simulated by using the control equation for solute diffusion of Fick's law.

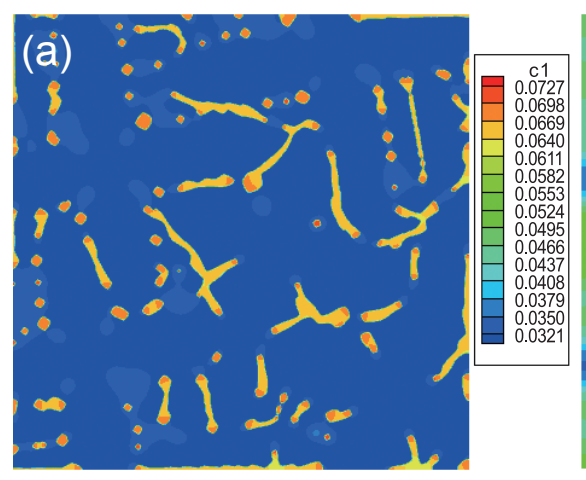

$0 \Delta t_{12}$

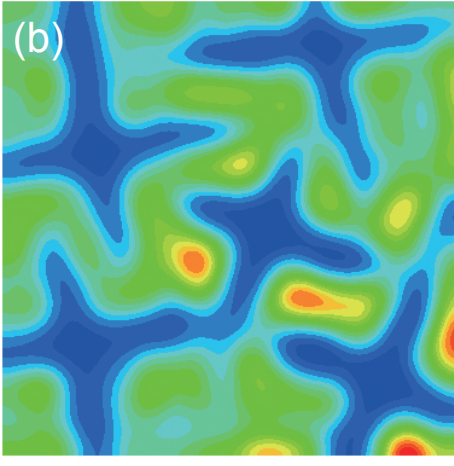

$200,000 \Delta t_{12}$

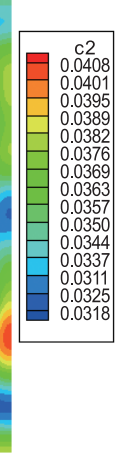

Fig. 3: Simulation results of solute field in liquid-solid phase solidification

\subsection{Simulation of eutectoid nucleation}

During the multi-phase transformation process of $\mathrm{Fe}-\mathrm{C}$ alloy, as the liquid-solid phase transformation ends, the temperature in the simulated region decreases. When reaching the eutectoid transformation temperature, the pearlite begins to nucleate. The simulation results of the system free energy change during the liquid-solid phase transition at the time of $0 \Delta t_{12}, 200,000 \Delta t_{12}$, and $400,000 \Delta t_{12}$, are shown in Fig. 4 , where the free energy at the austenite boundary is the highest. The thermal activation energy that the atoms in the parent phase have to overcome to enter the new phase is reduced ${ }^{[2]}$. Therefore, there is a great critical nucleation work at the grain boundary where is easy to nucleate. Liu et al. ${ }^{[16]}$ proposed that the composition of undercooled austenite is uneven under a certain degree of undercooling, and the carbon-rich and carbon-poor regions will fluctuate, which enhances the impact of structure and energy fluctuations. Once the nucleation conditions are reached, the cementite will nucleate in the carbon-rich region while the ferrite will nucleate in the carbon-poor region. The cementite and ferrite are nucleated simultaneously, and jointly create a pearlite nucleus $\left(\mathrm{F}+\mathrm{Fe}_{3} \mathrm{C}\right)$. Therefore, when the pearlite nucleation is set in the simulation work, there is no leading phase of cementite or ferrite. According to the general rule of solid phase transformation, the austenite grain boundary is the place where pearlite preferentially nucleates, because the grain boundary energy is high, the carbon atoms are more concentrated and irregularly, which are favourable for nucleation ${ }^{[17]}$.

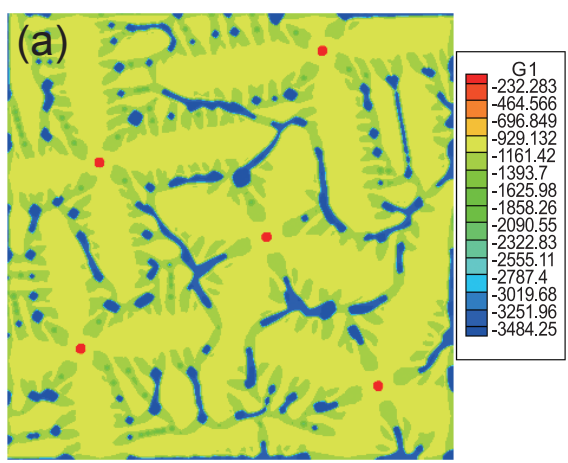

$0 \Delta t_{12}$

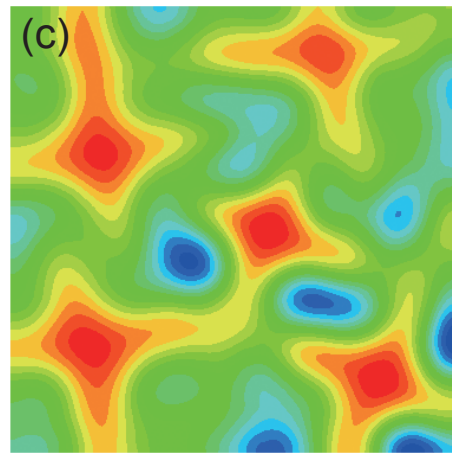

$400,000 \Delta t_{12}$

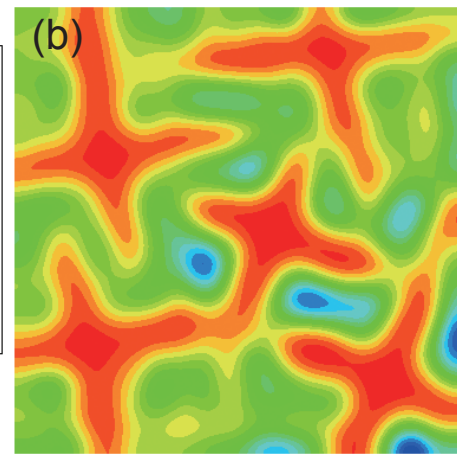

$200,000 \Delta t_{12}$

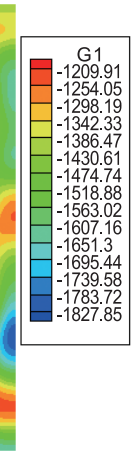

Fig. 4: Simulation results of free energy in liquid-solid phase transformation
According to the calculation of the nucleation model in Section 2.4, the nucleation site of the eutectoid structure in the continuous phase transformation is marked by the black circles in Fig. 5(a). The critical nucleation energy at grain boundaries is high, which makes the boundaries easy for nucleation. The nucleation phenomenon in Fig. 5(a) and Fig. 6(a) are consistent with the nucleation theory of Cahn ${ }^{[18]}$. The simulation result is consistent with the results of Liu et al. ${ }^{[19]}$, i.e., the pearlite nucleates at grains boundary, as shown in Fig. 5(b).

\subsection{Simulation results of pearlite growth and analysis}

Figure 6 shows the simulation results of the pearlites that grown in a diffusive shared-steps mechanism. Figure 6(a) shows the simulation results of the pearlite nucleation sites when the austenite in Fig. 1(c) fills the simulation area and reaches the nucleation condition. In order to observe the growth process of pearlite in austenite grains with different morphologies more easily, the austenite is marked with different colours in Fig. 6(a). 

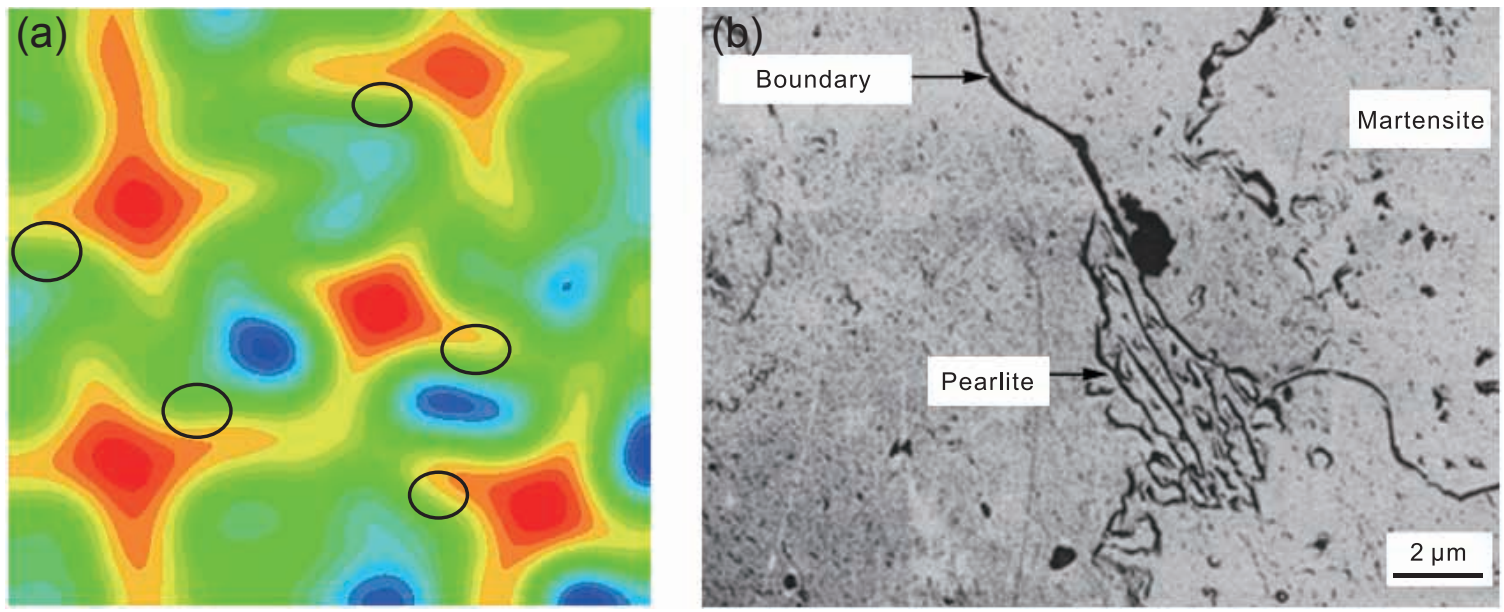

Fig. 5: Nucleation site of pearlite at grains boundary ${ }^{[19]}$
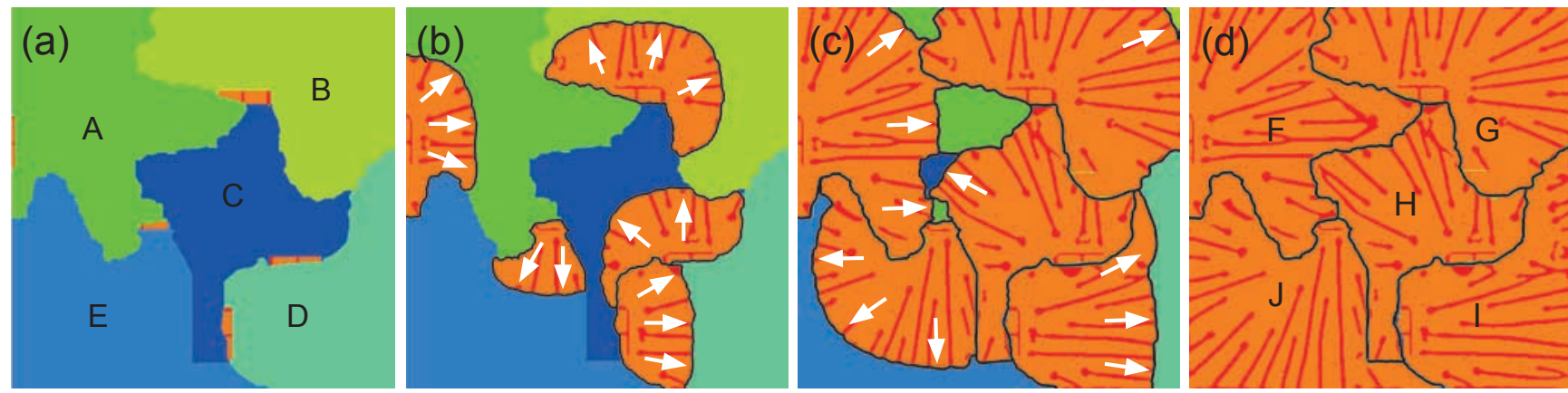

Fig. 6: Simulation results of morphology of pearlite

Meanwhile, the growth boundary of pearlite is marked with black solid lines, so that the morphology of pearlite in different parent phases can be observed conveniently.

It can be seen from Fig. 6 that the pearlite grows toward the interior of austenite grains, and the eutectoid structure at the front of the pearlite grows in a bifurcated way. The direction of the bifurcation growth is shown by the arrows in Figs. 6(b) and (c). The front of the pearlite grows in a spherical crown shape, and finally forms a lamellar pearlite structure, as shown in Fig. 6(d). The simulation results are consistent with the results obtained by Cong et al. ${ }^{[20]}$, as shown in Fig. 7, and the white arrow in Fig. 7 indicates the bifurcation growth direction of pearlite.

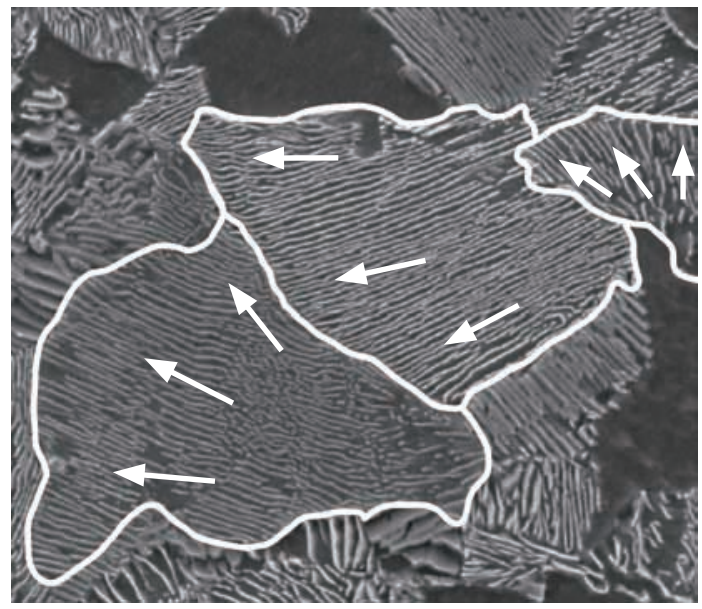

Fig. 7: Morphology of lamellar pearlite ${ }^{[20]}$
When the pearlite nucleates at the austenite grain boundary, the $\mathrm{Fe}_{3} \mathrm{C}$ in pearlite and adjacent austenite grains keeps a certain crystallographic orientation. Additionally, the relationship between ferrite in pearlite and austenite keeps K-S relationship ${ }^{[21]}$. Pearlite nucleates at the austenite grain boundaries and grows toward the interior of the adjacent austenite at the noncoherent interfaces, rather than toward the austenite at coherent interface. This is because the pearlite has a greater mobility in the austenite at the non-coherent interface ${ }^{[22,23]}$. For example, the pearlite grows into the A grain of austenite in a shared-step mechanism after nucleation at grain boundaries of Austenites $\mathrm{A}$ and $\mathrm{E}$. In addition, during the simulation, it was also found that the austenite has a hereditary influence on the pearlite, the morphology of the pearlite was close to that the parent austenite grains, as shown in Fig. 6(d).

Different nucleation sites have certain influence on the growth rate of pearlite. Figure 8 is the transformation curve of parent austenite corresponding to Fig. 6, in which the slope of the curve represents the growth rate of pearlite. It can be seen that the transformation rate of austenite increases slowly with the change of time when eutectoid transformation occurs in austenite. However, when the transformation time exceeds 50,000 $\Delta t_{2}$, the rate of transformation gradually decreases until the eutectoid transformation is completed. The Pearlite $\mathrm{G}$ and $\mathrm{H}$ are nucleated at the convex boundary of the parent phase, as shown in Fig. 6(a). When the time approaching 70,000 $\Delta t_{2}$, the transformation of austenite has come to an end, and the transformation curves $\mathrm{B}$ and $\mathrm{C}$ are shown in Fig. 8. The Pearlite $\mathrm{F}$ and $\mathrm{I}$ are nucleated 


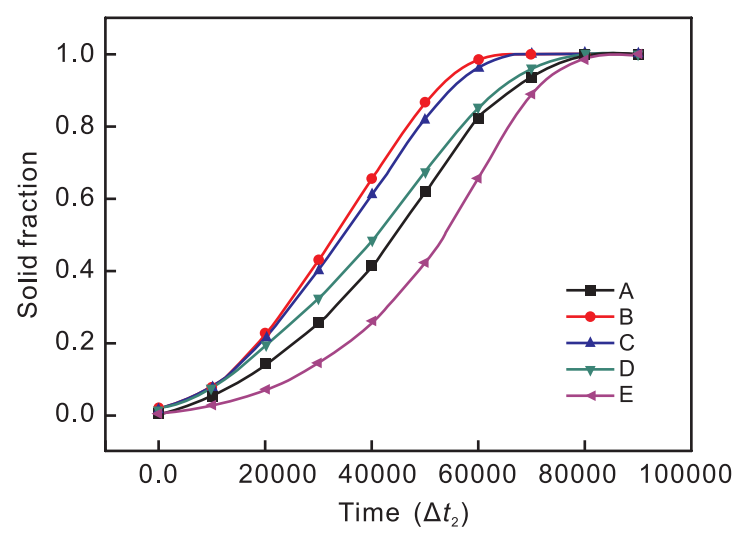

Fig. 8: Relationship between austenite transformation and time

at the concave boundary of parent phase, as shown in Fig. 6(a). The nucleus of Pearlite $\mathrm{J}$ are nucleated at the concave boundary of parent phase. When the time is close to $90,000 \Delta t_{2}$, the transformation of austenite has come to an end, and the transformation curves D and E are shown in Fig. 8. The nucleus of Pearlite F and I is located at the flat boundary. When the time is close to $80,000 \Delta t_{2}$, the transformation of austenite has come to an end. This indicates that when the pearlite nucleates at the grain boundary, the growth rate of pearlite at the convex boundary is faster than that at the concave boundary.

\section{Conclusions}

This study established a multi-phase-field model of liquidsolid-eutectoid continuous phase transformation. Taking Fe-C alloy as an example, the microstructural evolution process of continuous phase transformation of alloys was simulated, and the simulation results are as follows:

(1) Temperature function $K$ was used to control the sequence of phase transformation. The multi-phase-field model of liquidsolid-eutectoid continuous phase transformation can accurately simulate the multi-phase transformation process of $\mathrm{Fe}-\mathrm{C}$ alloy.

(2) As the liquid-solid phase transformation ends, the pearlite begins to nucleate when the eutectoid nucleation conditions are reached. After nucleation on the boundaries of austenite grains, the pearlite grows toward the interior of austenite grains, and the eutectoid structure at the front of the pearlite grows in a bifurcate manner. The austenite structure has hereditary effect on the pearlite, and the morphology of pearlite structure is similar to the parent austenite grain. The growth rate of pearlite is related to the site of pearlite nucleation, when the pearlite nucleates at the convex boundaries, it has a faster growth rate.

\section{Acknowledgements}

This work was financially supported by the National Natural Science Foundation of China (Grant Nos.: 11504149, 51661020) and the Natural Science Foundation of Gansu Province of China (Grant No.: 18JR3RA147).

\section{References}

[1] Liu J K, Niu Y N, Yan W, et al. Simulation of grain growth by phase field method. Journal of Xi 'an University of Technology, 2008, 28(4): 360-365. (In Chinese)

[2] Cui $Z$ Q. Metallology and heat treatment principle. Beijing: Machinery Industry Press, 2007: 241. (In Chinese)

[3] Zhao Y H, Cheng J, Hou H, et al. Progress in numerical simulation of microstructure during solidification. Journal of North University of China, 2006, 27(4): 372-376. (In Chinese)

[4] Kang Y S, Zhao Y H, Hou H, et al. Simulation of liquid channel of Fe-C alloy directional solidification by phase-field method. Acta Physica Sinica, 2016, 65(18): 308-314. (In Chinese)

[5] Tourret D, Karma A. Growth competition of columnar dendritic grains: A phase-field study. Acta Materialia, 2015, 82: 64-83.

[6] Feng L, Lu N N, Gao Y L, et al. Phase-field simulation of secondary dendrite growth in directional solidification of binary alloys. China Foundry, 2019, 16(2): 97-104.

[7] Zhu C S, Xiao R Z, Wang Z P, et al. Numerical simulation of recalescence of 3-dimensional isothermal solidification for binary alloy using phase-field approach. Transactions of Nonferrous Metals Society of China, 2009, 19(5): 1286-1293.

[8] Zhang A, Meng S X, Guo Z P, et al. Dendritic growth under natural and forced convection in Al-Cu alloys: From equiaxed to columnar dendrites and from $2 \mathrm{D}$ to $3 \mathrm{D}$ phase-field simulations. Metallurgical and Materials Transactions B, 2019, 50(3): 1514-1526.

[9] Zhang A, Guo Z P, Xiong S M. Phase-field-lattice Boltzmann study for lamellar eutectic growth in a natural convection melt. China Foundry, 2017, 14 (5): 373-378.

[10] Zhang J, Chen W X, Zheng C W, et al. Phase field simulation of austenite-ferrite phase transition in Fe-C-Mn ternary alloy. Acta Metallurgica Sinica, 2017, 53(6): 760-768. (In Chinese)

[11] Zhang J, Chen W X, Zheng C W, et al. Phase-field modeling of ferrite morphology in austenite-to-ferrite transformation with considering anisotropic effects. Acta Physica Sinica, 2017 (7): 282-291. (In Chinese)

[12] Nakajima K, Apel M, Steinbach I. The role of carbon diffusion in ferrite on the kinetics of cooperative growth of pearlite: A multiphase field study. Acta Materialia, 2006, 54(14): 3665-3672.

[13] Han Z Q, Shen H F, Xiong S M, et al. Numerical simulation on fluid, heat and mass transfer in solidification of Fe-C alloys. Acta Metallurgica Sinica, 2002, 38(9): 941-946. (In Chinese)

[14] Kim S G, Kim W T, Suzuki T, et al. Phase-field modeling of eutectic solidification. Journal of Crystal Growth, 2004, 261(1): 135-158.

[15] Steinbach I, Apel M. Multi phase field model for solid state transformation with elastic strain. Physica D: Nonlinear Phenomena, 2006, 217(2): 153-160.

[16] Liu Z C, Ren H P. Diffusion-type transformation of undercooled austenite. Beijing: Science Press, 2007: 21-24. (In Chinese)

[17] Liu Z C, Ren H P, Wang H Y. Austenite formation and pearlite transformation. Beijing: Metallurgical Industry Press, 2010: 55-59. (In Chinese)

[18] Cahn J W. The kinetics of grain boundary nucleated reactions. Acta Metallurgica, 1956, 4(5): 449-459.

[19] Liu Z C. Recent evolution of pearlite transformation theory. Metal Heat Treatment, 2008(4): 6-13. (In Chinese)

[20] Cong T, Han J M, Chen G, et al. Study on performance of new material wheels for high speed electric multiple units. China Railway Science, 2018, 39(1): 75-81. (In Chinese)

[21] Qi Z F. Diffusion and phase transition in solid metals. Beijing: Machinery Industry Press, 1998: 133-140. (In Chinese)

[22] Li F, Gao Y L, Lu N N, et al. Phase-field simulation of competitive growth of grains in a binary alloy during directional solidification. China Foundry, 2018, 15(5): 333-342.

[23] Jhon D V, Lu G X, Zhao Z W. Fundamentals of physical metallurgy. Shanghai: Science and Technology Press, 1980: 260-265. (In Chinese) 